\title{
RADIOMETRIC BLOCK ADJUSMENT AND DIGITAL RADIOMETRIC MODEL GENERATION
}

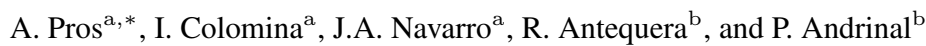 \\ ${ }^{\text {a } I n s t i t u t e ~ o f ~ G e o m a t i c s, ~ A v . ~ C a r l ~ F r i e d r i c h ~ G a u s s, ~} 11$ - Parc Mediterrani de la Tecnologia, 08860 Castelldefels, Spain - \\ (alba.pros, iael.colomina, jose.navarro)@ideg.es \\ ${ }^{\mathrm{b}}$ Altais SL, Juan Alvarez Mendizabal, 76 Bajo C, 28008 Madrid, Spain - \\ (r.antequera, p.andrinal)@altais-sl.com
}

\section{Commission WG VI/4}

KEY WORDS: Radiometry, photogrammetry, digital terrain model, radiometric terrain response, radiometric calibration, block adjustment, atmospheric models, BRDF

\begin{abstract}
:
In this paper we present a radiometric block adjustment method that is related to geometric block adjustment and to the concept of a terrain Digital Radiometric Model (DRM) as a complement to the terrain digital elevation and surface models. A DRM, in our concept, is a function that for each ground point returns a reflectance value and a Bidirectional Reflectance Distribution Function (BRDF). In a similar way to the terrain geometric reconstruction procedure, given an image block of some terrain area, we split the DRM generation in two phases: radiometric block adjustment and DRM generation. In the paper we concentrate on the radiometric block adjustment step, but we also describe a preliminary DRM generator. In the block adjustment step, after a radiometric pre-calibraton step, local atmosphere radiative transfer parameters, and ground reflectances and BRDFs at the radiometric tie points are estimated. This radiometric block adjustment is based on atmospheric radiative transfer (ART) models, pre-selected BRDF models and radiometric ground control points. The proposed concept is implemented and applied in an experimental campaign, and the obtained results are presented. The DRM and orthophoto mosaics are generated showing no radiometric differences at the seam lines.
\end{abstract}

\section{INTRODUCTION}

In this paper we present a radiometric block adjustment method that is related to geometric block adjustment and to the concept of a terrain Digital Radiometric Model (DRM) as a complement to the terrain digital elevation and terrain models. A DRM is digital model that gives the reflectance value and the Bidirectional Reflectance Distribution Function (BRDF) of each ground point.

In the block adjustment step, the camera is radiometrically precalibrated, some local atmosphere radiative transfer parameters and the reflectance and BRDF are estimated at the radiometric tie points. This radiometric block adjustment is based on radiative transfer models, pre-selected BRDF models and radiometric ground control points.

The underlying concept is that if a Digital Terrain Model (DTM) and/or Digital Elevation Model (DEM) together with a DRM are available, not only the traditional cartographic representations of the terrain can be produced, but also realistic simulations of an area can be produced by freely setting parameters like the time (date and time within the day) and the atmospheric conditions.

The importance of radiometry in the digital airbone photogrammetry, in comparison to the analog systems is highlighted by (Honkavaara et al., 2009). Besides, (Honkavaara et al., 2009) point that improving the automation potential of photogrammetric applications is possible with a rigorous treatment of image radiometry.

The knowledge of the terrain radiometric response (DRM) also allows correcting all the images of the whole block eliminating the radiometric differences between them. The correction of the radiometry in aerial images is one of the main milestones in aerial photogrammetry in order to improve their applications such as otrhophoto mosaics or land-cover classification.
In the related literature different ideas to correct the radiometric heterogeneities are available.

A radiometric aerial triangulation is presented by (Chandelier and Martinoty, 2009), with analogy to the standard aerial triangulation to generate orthoimages without radiometric heterogeneities. Instead of considering physically-based models, they propose another category of radiometric correction methods: empirical (statistical approach based on histogram equalization) or semi-empirical (taking into account only the most prominent radiometric effects (for examples BRDF or the atmospheric model proposed by (Richter and Schläpfer, 2002))) corrections. They provide a relative correction between images, using exclusively image-based information.

A polynomial radiometric block adjustment is proposed by (Falala et al., 2008). Similar to the approach presented by (Chandelier and Martinoty, 2009), (Falala et al., 2008) do not use a physical model.

(Olsen et al., 2010) suggest a method for the calibration of agricultural cameras (AgCam). A radiometric block adjustment is proposed for correcting the vignetting effect, the Charge-Coupled Device (CCD) non-uniform quantum efficiency and the ChargeCoupled Device (CCD) dark current, separately. At the end, by calibrating the AgCams and correcting all the images, they reduce the radiometric heterogeneities in the orthophoto mosaics.

A radiometric correction process for UAV image blocks based on a radiometric block adjustment is proposed by (Honkavaara et al., 2012a). They propose a physical model taking into account all the elementary components of radiance entering into a sensor in a UAV flight and an empirical model for the computation of the digital number from the reflectance value, considering the $\mathrm{Bi}$ directional reflectance factor of each object. 
In this paper we propose and test a radiometric block adjustment concept. In a way, it is similar to those previously reported by (Chandelier and Martinoty, 2009), (Richter and Schläpfer, 2002), (Falala et al., 2008) and (Olsen et al., 2010), but using physicaloriented models, like (Honkavaara et al., 2012a). However, the physical-oriented models used in the present paper are different than the one used by (Honkavaara et al., 2012a). In our concept, after a radiometric pre-calibration (vignetting effect) step we perform a self-calibrating radiometric block adjustment with radiometric calibration, BRDF and atmospheric radiative transfer (ART) models. In the adjustment, the observations are the image digital numbers (DN) and the ground control reflectances $(\rho)$. The unknown parameters are the sensor calibration parameters, the ground reflectances of the radiometric tie points and additional BRDF and ART parameters. From these parameters, both the DRM and the radiometrically corrected images can be derived. The orthophoto mosaics obtained with the corrected images will then allow to at least verify the consistency of the radiometric adjustment results.

In the following section the radiometric camera calibration models, the atmospheric model and the BRDF model are described, as well as the method for the radiometric block adjustment. In section 3, we present the DRM generation concept. An experimental campaign to validate the proposed approach is detailed in section 4. Finally, some conclusions are presented as well as further research to be pursued.

\section{RADIOMETRIC MODELS AND BLOCK ADJUSTMENT}

The radiometric calibration and block adjustment procedure is divided in two phases: pre-calibration and self-calibrating block adjustment. As mentioned, the model for the radiometric measurements (DNs) includes a radiometric self-calibration model, a BRDF model and an atmospheric radiative transfer (ART) one.

\subsection{Radiometric pre-calibration}

The pre-calibration model proposed in this paper aims at correcting the vignetting effect, which consists of the radial falloff of the intensity from the principal point of the image, as detailed by (Olsen et al., 2010), (Goldman, 2010) and (Zheng et al., 2009). The vignetting effect can be produced by different physical causes: natural vignetting (due to geometric optics: angle at which the light exits from the rear of the lents), pixel vignetting (due to the angular sensitivity of digital optics), optical vignetting (due to the light path blocked inside the lens body by the lens diaphragm, easily observed by the changing shape of the clear aperture of the lens, which reduces the amount of light reaching the image plane) and mechanical vignetting (due to certain light path becoming blocked by other camera elements). However, a single "elliptic" polynomial estimation model is sufficient to describe the phenomena. Given a reference calibration object surface with homogeneous constant $d n_{0}$ we have used the 6th degree polynomial

$$
d n_{0}-\left(1+v_{1} r^{2}+v_{2} r^{4}+v_{3} r^{6}\right)\left(d n+v_{d}\right)=0
$$

with

$$
r=\sqrt{\frac{\left(x-\left(x_{0}+\Delta x_{0}\right)\right)^{2}}{k_{1}^{2}}+\frac{\left(y-\left(y_{0}+\Delta y_{0}\right)\right)^{2}}{k_{2}^{2}}} .
$$

$d n$, the measurement, is the digital number of an image pixel and $v_{n}$ its residual. $\left(x_{0}, y_{0}\right)$ is the principal point of symmetry (PPS) of the image. Besides, $\left(\Delta x_{0}, \Delta y_{0}\right)$ model the vignetting decentering with respect to the PPS and $k_{1}$ and $k_{2}$ stand for the major and minor semi-axis of the "ellipse."

We have found that a global calibration parameter subset $\Delta x_{0}$, $\Delta y_{0}, k_{1}$ and $k_{2}$ suffices while colour dependent subsets $v_{1}, v_{2}$, $v_{3}$ are required for each colour channel.

\subsection{Radiometric self-calibration}

The self-calibration phase estimates the gain $(\alpha)$ and offset $(\beta)$ of the camera, giving the relation between the at-sensor radiance $\left(L_{S}\right)$ and the digital number $(d n)$ for each pixel:

$$
d n=\alpha L_{S}+\beta .
$$

To a large extent, the values of $\alpha$ and $\beta$ are constant for all the images taken with the same sensor within some period of time. However, theses values are different for each colour channel.

\subsection{Atmospheric radiative transfer model}

In this work, the proposed atmospheric radiative transfer model is based on the Radiative Transfer Model (RTM) used by (Beisl et al., 2008). The RTM takes into account the solar irradiance, the diffuse radiation, the backscattered solar irradiance and the solar radiance reflected by the surroundings:

$$
L_{S}=L_{0}+\frac{\rho \rho_{i} S T_{d} T_{u}}{\pi\left(1-s \rho \rho_{i}\right)}
$$

with $L_{0}$ standing for the upward radiance of the atmosphere for zero surface reflectance.

$$
S=S_{0}\left(\frac{a}{r}\right)^{2} \cos \theta_{i}
$$

with $S_{0}=1.36 \cdot 10^{3} \mathrm{~W} / \mathrm{m}^{2}$ being the mean solar irradiance, $a$ the Earth-Sun distance, $r$ the mean Earth-Sun distance, and $\theta_{i}$ the Sun zenith angle. $\rho_{i}$ is the reflectance of one single point in the terrain and $\rho$ corresponds to its Bidirectional Reflectance Distribution Function correction (described in section 2.4). $T_{u}$ is the total upward transmittance from ground to the sensor, $T_{d}$ is the total downward transmittance from the Sun to the ground and $s$ is the albedo, which is defined as the fraction of the upward radiance which is backscattered by the atmosphere.

Figure 1 depicts the phenomena contributing to the RTM, from the solar irradiance to the at sensor radiance measured by the sensor, taking into account the reflected radiance by the terrain point. The influence of the solar elevation in the proposed model is accounted for the value of $\theta_{i}$. A comprehensive study about the influence of the solar elevation both in radiometric and in geometric performance of digital airbone photogrammetry is presented by (Honkavaara et al., 2012b).

\subsection{Bidirectional Reflectance Distribution Function}

The radiometric model includes the Bidirectional Reflectance Distribution Function (BRDF) proposed in (Beisl and Woodhouse, 2004) which is based on Sun position, sensor orientation and terrain surface parameters $(a, b, c, d, e)$. Thus, the terrain has to be classified a priori depending on its reflectance properties. The Beisl-Woodhouse BRDF is given by

$$
\begin{aligned}
& \rho\left(\theta_{i}, \theta_{r}, \varphi, a, b, c, d, e\right)= \\
& \quad a \theta_{i}^{2} \theta_{r}^{2}+b\left(\theta_{i}^{2}+\theta_{r}^{2}\right)+c \theta_{i} \theta_{r} \cos \varphi+ \\
& \quad d \sqrt{\tan ^{2} \theta_{i}+\tan ^{2} \theta_{r}-2 \tan \theta_{i} \tan \theta_{r} \cos \varphi}+e
\end{aligned}
$$

where $\theta_{i}$ is the incident illumination zenith angle, $\theta_{r}$ stands for the reflexion view zenith angle and $\varphi$ is the relative azimuth angle (Figure 2). 


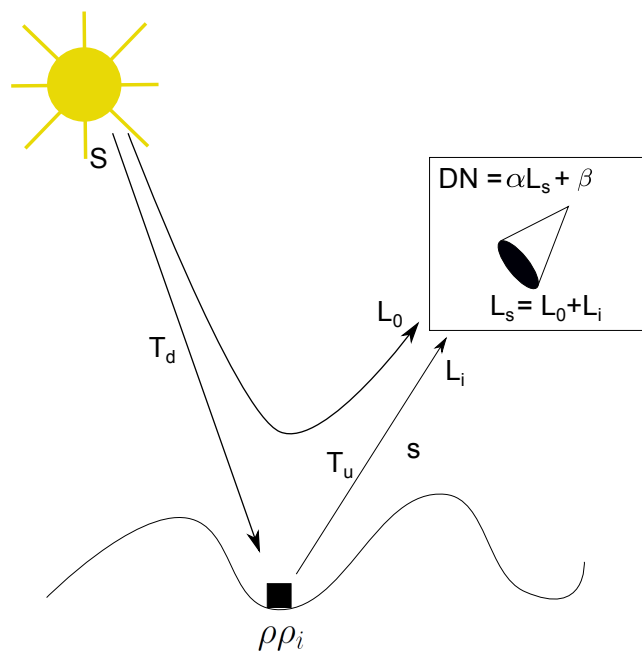

Figure 1: RTM scheme.

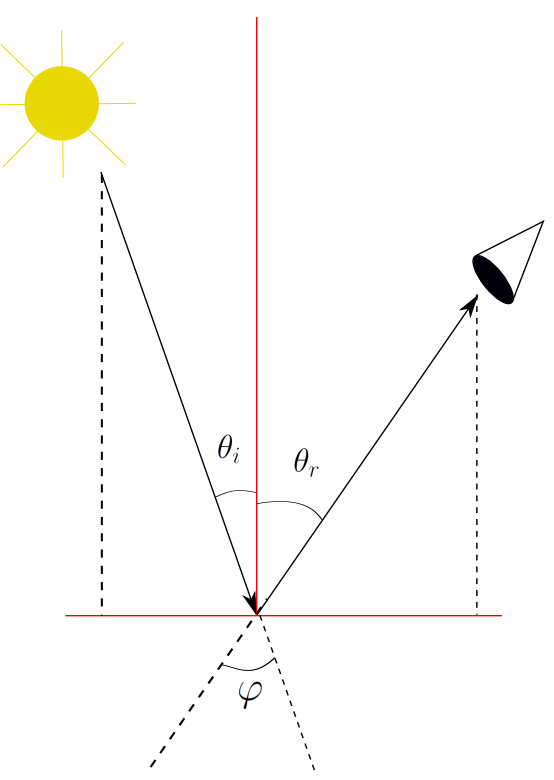

Figure 2: BRDF angles.

\subsection{Radiometric block adjustment}

The camera calibration model, the BRDF model and the atmospheric RTM are integrated in the observation equation 7 for the radiometric block adjustment. We have followed a similar procedure as in the standard "geometric" aerial triangulation, that is, a radiometric block adjustment with radiometric tie and control points.

The radiometric control points are ground points with measured reflectance (different for each channel: red, green, blue) and whose ground coordinates are known.

The radiometric tie points are derived from the geometric tie points by identifying near by points whose local area ( $10 \times 10$ pixels) exhibits an homogeneous colour intensity. Once the radiometric tie point is chosen, the translation between this point and the geometric tie point is applied to all the images in which the geometric tie point was in order to get the position of this radiometric tie point in each image.

Considering equations $1,3,4$ and 6 , the problem to be solved in the radiometric block adjustment is to minimize the residuals $\left(v_{i j}\right)$ of the following equation:

$$
D N_{i j}+v_{i j}=a_{r}\left(a_{a}\left(L_{0}+\frac{\rho \rho_{i} S T^{2}}{\pi\left(1-s \rho \rho_{i}\right)}\right)+b_{a}\right)+b_{r} .
$$

$v_{i j}$ is the residual of the observation $D N_{i j}$ at the radiometric tie point $i$ in the image $j . a_{a}$ and $b_{a}$ correspond to the offset and gain of the sensor for the whole block, while $a_{r}$ and $b_{r}$ stand for the offset and gain for each image of the block.

For the radiometric control points, we use the usual pseudo-observation equation

$$
l_{\rho_{i}}+v_{\rho_{i}}=\rho_{i}
$$

being $l_{\rho_{i}}$ the reflectance value measured for a radiometric control point, $v_{\rho}$ its residual and $\rho_{i}$ the unknown reflectance parameter.

The parameters to be estimated are $a_{a}, b_{a}, L_{0}, T$ and $s$ for the whole block, $a_{r}$ and $b_{r}$ for each image, $a, b, c, d$ and $e$ for each terrain group and $\rho_{i}$ for each tie point. In this particular research and preliminary testing we "stabilized/weighted" the image dependent $a_{r}$ and $b_{r}$ parameters to their "normal" values 1 and 0 respectively as the radiometric network strength is not sufficiently strong.

The above models were coded and implemented as $\mathrm{C}++$ classes of a "radiometric toolbox" that run on the generic network adjustment platform GENA (Colomina et al., 2012).

\section{DRM GENERATION}

The Digital Radiometric Model (DRM) is, as mentioned, a digital model which, for each ground point, gives its reflectance value and the corresponding BRDF.

Using the DTM, given the digital values of the images and the estimated parameters obtained from the radiometric block adjustment, the reflectance of each ground point is estimated. Moreover, the BRDF of the ground points is also computed. Ideally, one should have the BRDF parameters classified as a function of the point ground coordinates. However, in the case of homogeneous areas, the BRDF parameters can be assumed to be constant. Thus, with the reflectance values and the BRDF of each ground point, the DRM of the block is generated. 
International Archives of the Photogrammetry, Remote Sensing and Spatial Information Sciences, Volume XL-1/W1, ISPRS Hannover Workshop 2013, 21 - 24 May 2013, Hannover, Germany

\begin{tabular}{l|rr} 
Parameter & $\sigma$ & \multicolumn{1}{|c}{ Units } \\
\hline$\rho_{i}$ & 0.0192 & - \\
$a_{a}$ & 0.0013 & $\mathrm{rad}^{-2} \mathrm{~m}^{2} W^{-1}$ \\
$b_{a}$ & 0.0013 & - \\
$L_{0}$ & 0.0044 & $\mathrm{Wrad}^{2} \mathrm{~m}^{-2}$ \\
$T$ & 0.0003 & - \\
$s$ & 0.0025 & $\mathrm{rad}^{-2}$ \\
$a$ & 0.0131 & $\mathrm{rad}^{-2}$ \\
$b$ & 0.0129 & - \\
$c$ & 0.0131 & $\mathrm{rad}^{2}$ \\
$d$ & 0.0128 & $\mathrm{rad}^{2}$ \\
$e$ & 0.0130 &
\end{tabular}

Table 1: Radiometric block adjustment: precision of results.

For any image of the block, for each point $i$, given the colour intensity $\left(d n_{i}\right)$ and considering all the adjusted parameters, its reflectance $\left(\rho_{i}\right)$ is computed, for each channel as follows:

$$
d n_{i} \longrightarrow A_{i} \longrightarrow \rho_{i}
$$

with

$$
A_{i}=\frac{\frac{d n_{i}-b_{r}}{a_{r}}-b_{a}}{a_{a}}-L_{0}
$$

and

$$
\rho_{i}=\frac{A_{i} \pi}{\rho\left(A_{i} \pi s+S T^{2}\right)}
$$

From the DRM it is now easy to generate both continuous orthophotos and "radiometrically corrected" images for arbitrary Sun and sensor position and orientation. For example, one can assume that the total upward transmittance from ground to the sensor and the total downward transmittance from the Sun to the ground is the whole solar irradiance, that is, $T=1$; further, that there is no fraction of the upward radiance which is backscattered by the atmosphere, thus, $s=0$; and finally, that the Sun and the sensor are oriented in a way such that $\theta_{i}=\theta_{r}=\phi=0$.

The corrected intensity colour value $\left(d n_{i}^{c}\right)$ is computed then as

$$
d n_{i}^{c}=\frac{\rho_{0} \rho_{i} S_{0}}{\pi}
$$

being $S_{0}=S$ (equation 5) and $\rho_{0}=\rho$ (equation 6) assuming that $\theta_{i}=\theta_{r}=\phi=0$.

\section{EXPERIMENTAL CAMPAIGN}

In order to validate the proposed approach, we present the preliminary results for a photogrammetric flight over Extremadura (Spain) with the UltraCam Xp-WA camera.

\subsection{Photogrammetric data acquisition}

In June and July 2010, a fligth over an area of 750000 Ha was performed in the NW of Extremadura (Spain). While the flight was taking place, 5 groups of 6 radiometric ground control points (RGCP) per group were measured. 4 groups were located at the block corners and 1 at the block centre. Each radiometric control point consisted of a $2 \times 2 \mathrm{~m}^{2}$ radiometric target. Within a group six colours were used, one per RGCP: red, green, blue, gray, white and black. For each RGCP both the reflectance and the radiance was measured with the ASD FieldSpec 3 spectroradiometer. The Ground Sampling Distance (GSD) is approximately $45 \mathrm{~cm}$ and forward- and cross-overlap is of $60 \%$ and $30 \%$, respectively.

\begin{tabular}{l|cccr} 
Type & $\mu$ & RMS & $\sigma$ & Units \\
\hline tie & -.0002 & 0.038 & 0.038 & DN [0,1] \\
check & 0.0246 & 0.169 & 0.167 & -
\end{tabular}

Table 2: Residuals (radiometric tie points) and differences (radiometric check points).

The camera used is the UltraCam Xp Wide Angle S/N UC-SXpwa-50814031, a frame camera with multispectral capacity. The sensor unit is composed of 8 cones (or independent cameras): 4 of them capture the panchromatic image while the other 4 generate the multispectral image.

From the aerial triangulation geometric tie points, the radiometric tie points were calculated as described in section 2.5. In addition, the image and ground coordinates of the RGCP were measured and computed respectively. All these, resulted in a radiometric block of 337 images, 4764 radiometric tie points and 21577 radiometric image measurements. For this preliminary analysis the 4 corner RCGP groups were used as actual control and the central RCGP was used for check purposes.

The precision of the radiometric image measurements (DN) normalized to the $[0,1]$ range is 0.03 and the RGCP were considered error free.

Last not least, we acknowledge that an important limitation of the data used was the post-processed nature of the images since we had no access to the raw image data.

\subsection{Block adjustment and preliminary results}

For the whole block, an homogeneous surface is assumed, thus, there are just three set of BRDF paraneters $a, b, c, d$, and $e$; one for each colour channel. As mentioned, $a_{r}$ and $b_{r}$ are assumed to be different for each image but stabilized around "normal" values 1,0 with $\sigma_{a_{r}}=\sigma_{b_{r}}=0.01$ due to correlations with $a_{a}$ and $b_{a}$. Since atmospheric conditions did not change much during the flights there is just one ART parameter set $T, s$ and $L_{0}$. Last, for each radiometric tie point and colour channel one parameter $\rho_{i}$ is to be estimated.

Tables 1 and 2, summarize the results obtained with the previously described models, data and settings. All estimated parameters are significant and within reasonable range of values. Residuals in the six RGCPs of the central group are reasonably low biased $(0.02[0,1]$-normalized $\mathrm{DN})$ altjough the dispersion $(0.16$ may seem too large). We will not enter a detailed discussion of these results as they are preliminary and, at this point in time of the research, the consistency and quality of the orthophoto mosaics is our main quality criteria.

\subsection{Quality of mosaics}

Given the obtained parameters in the radiometric block adjusment, and assuming standard atmospheric conditions the DRM is generated. That is, for each pixel of each image, given the original digital value $\left(d n_{i j}\right)$, its reflectance value is obtained (following equations 10 and 11). Finally, from the set of $\rho_{i}$-i.e., the DRM - and equation 12, radiometrically corrected images are obtained. Using comercially available SW, the DTM and the corrected images, radiometrically homgeneous othophotos were generated. We were then able to produce orthophoto mosaics without noticeable differences at the seam lines and to, according to end user empirical evaluations, produce more realistic orthophoto colours. 
International Archives of the Photogrammetry, Remote Sensing and Spatial Information Sciences, Volume XL-1/W1, ISPRS Hannover Workshop 2013, 21 - 24 May 2013, Hannover, Germany

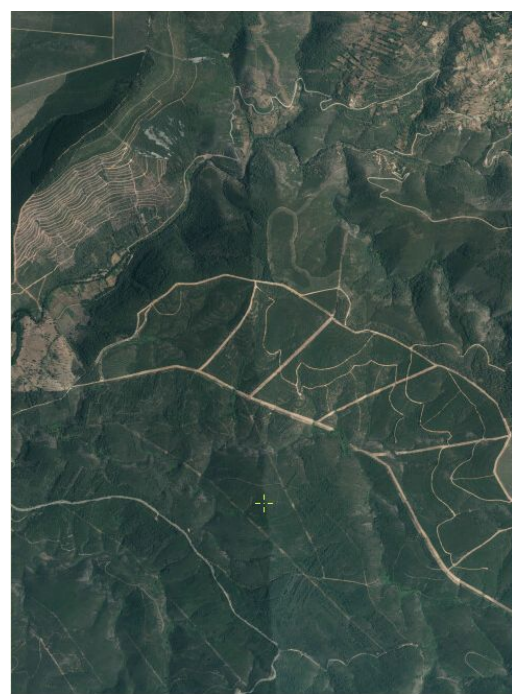

Figure 3: Original orthophoto mosaic (part 1)

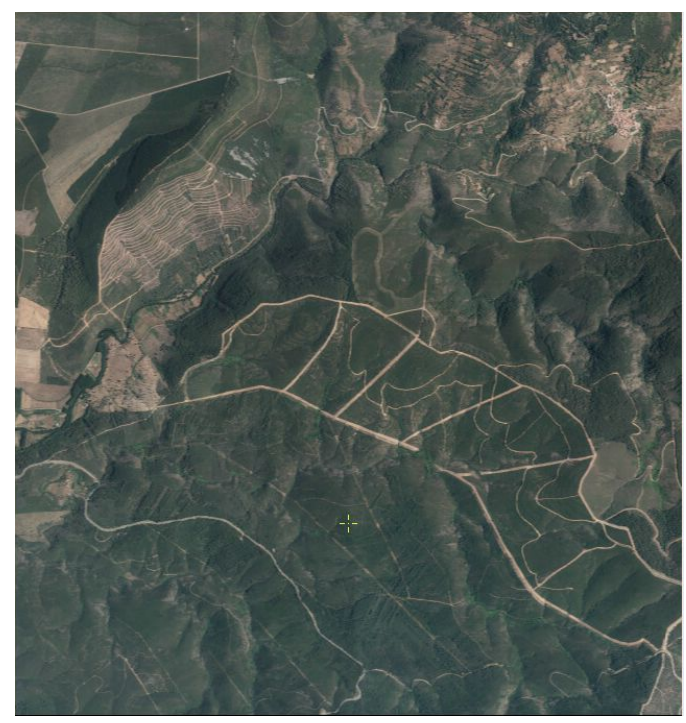

Figure 4: Corrected orthophoto mosaic (part 1)

In Figures 3 and 5 the orthophoto mosaics with original images are shown, while in Figures 4 and 6 the same orthophoto mosaics are presented, but with the radiometrically corrected images. Figures 3 and 4 correspond to one part of the DTM (part 1) and Figures 5 and 6 correspond to another part (part 2). Comparing both orthophoto mosaics, it is observed that the radiometric differences are largely reduced with the corrected images. According to local expertts, figures 4 and 6 , exhibit more realistic colours than figures 3 and 5 .

\section{CONCLUSIONS}

We have presented our preliminary modeling experiences and results of a radiometric block adjustment with the Beisl-Woodhouse BRDF and ART models. We have validated our results through radiometric check points and the inspection of seam lines of orthophoto mosaics.

We have introduced the concept of the Digital Radiometric Model for the terrain. DRMs of the Earth surface allow the generation of orthophotos with selectable Sun orientation, atmospheric conditions and sensor orientation. Actually, this is just a particular

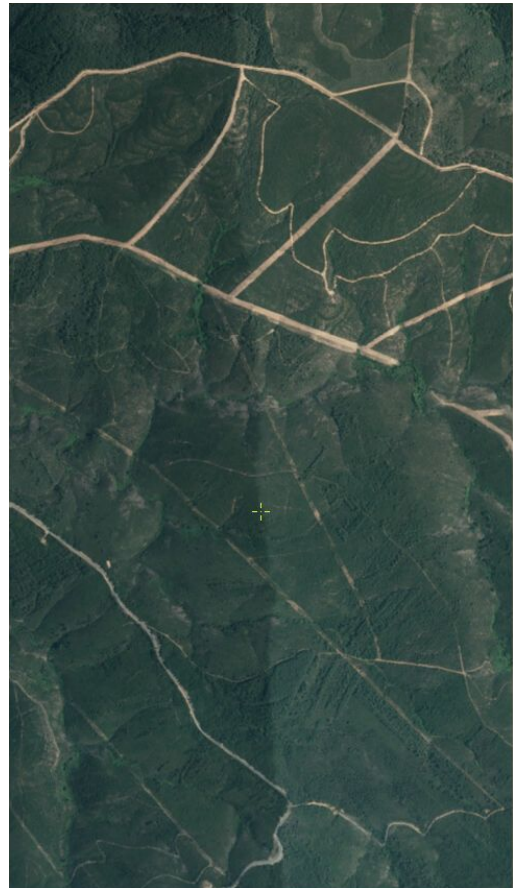

Figure 5: Original orthophoto mosaic (part 2)

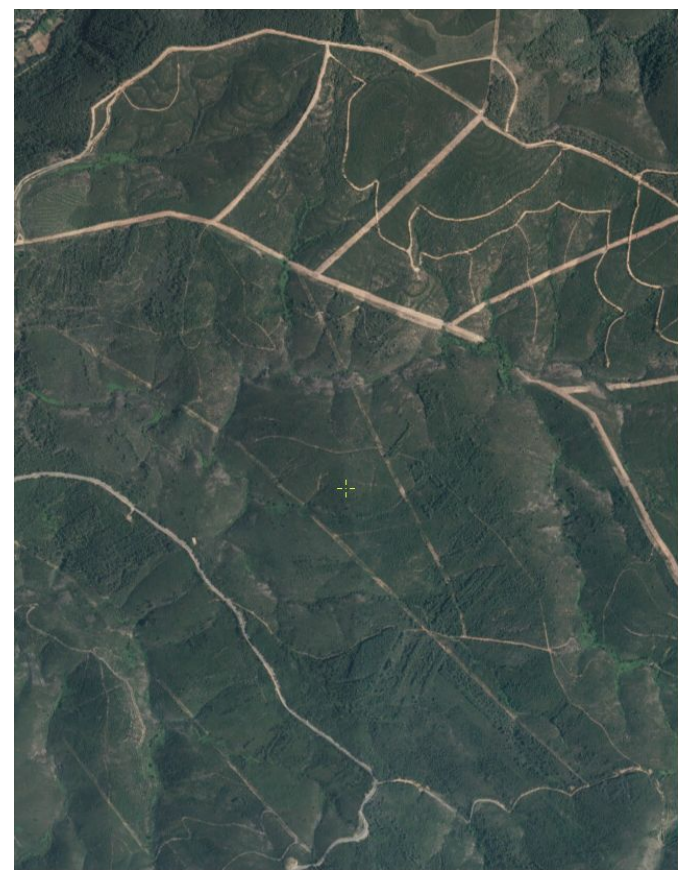

Figure 6: Corrected orthophoto mosaic (part 2) 
application of the DRM, since DRM allow to generate realistic views of the Earth surface for, as mentioned, selectable Sun, atmospheric and sensor parameters, Thus, with the knowledge of the DRM together with elevation models, not only the classical cartographic operations are feasible, but also $2 \mathrm{D}$ and $3 \mathrm{D}$ realistic simulations.

Although the materials used, namely the images from the Ultracam Xp Wide Angle S/N UC-SXp-wa-5081403 were not raw images and had already undergone commercial software corrections, the results were more than acceptable from the very beginning as shown by the residuals of the check points and by the unnoticeable mosaic seam lines. This indicates the robustness of the procedure probably due to the use of the radiometric control points. On the other side, we must acknowledge that the radiometric homogeneity of the test area have made things easy as we did not have to classify the terrain according to its reflectance properties.

\subsection{Further work}

The reported results are preliminary and a better understanding of the behaviour of the BRDF and ART models is required. Moreover, in the reported experimental campaign, a single set of BRDF parameters $a, b, c, d, e$ was estimated. For more practical and realistic realistic results, the terrain shall be classified into different surface types and even charecterize a priori some BRDF parameters. Thus, we will extend the method to inhomogeneous terrain areas and start with the usual four groups: asphalt, grass, water and sand. Use of the terrain inclinations is also planned. A last open point is the use of raw image data as opposed to the already pre-processed images. We plan on repeating the test if we get access to the raw images of the used Ultracam.

\section{ACKNOWLEDGEMENTS}

The research reported in this paper was carried out in the frame of the AUTORADCOR project that was partially funded by the "Centro para el Desarrollo Tecnológico Industrial" (CDTI), "Ministerio de Economía y Competitividad".

Revised April 2013

\section{REFERENCES}

Beisl, U. and Woodhouse, N., 2004. Correction of atmospheric and bidirectional effects in multispectral ads40 images for mapping purposes. Proc. XXth Congress of the ISPRS, Istanbul, Turkey p. 5.

Beisl, U., Telaar, J. and Schönermark, M. V., 2008. Atmospheric correction, reflectance calibration and brdf correction for ads 40 image data. The international archives of the photogrammetry, Remote sensing and spatial information science, Vol. XXXVII, Part B7, Beijing pp. 7-12.

Chandelier, L. and Martinoty, G., 2009. A radiometric aerial triangulation for the equalization of digital aerial images and orthoimages. Photogrannetric Engineering and Remote Sensing 75(2), pp. 193-200.

Colomina, I., Blázquez, M., Navarro, J. A. and Sastre, J., 2012. The need and keys for a new generation network adjustment software. International Archives of Photogrammetry, Remote Sensing and Spatial Information Sciences. 2012 August, September, Melbourne, Australia. pp. 303-308.
Falala, L., Gachet, R. and Cunin, L., 2008. Radiometric blockadjustment of satellite images reference $3 \mathrm{~d} \cap$ production line improvement. The international archives of the photogrammetry, Remote sensing and spatial information science, Vol. XXXVII, Part B4, Beijing pp. 319-323.

Goldman, D. B., 2010. Vignette and exposure calibration and compensation. Pattern Analysis and Machine Intelligence, IEEE Transactions on 32(12), pp. 2276-2288.

Honkavaara, E., Arbiol, R., Markelin, L., Martinez, L., Cramer, M., Bovet, S., Chandelier, L., Ilves, R., Klonus, S., Marshal, P., Shläpfer, D., Tabor, M., Thom, C. and Veje, N., 2009. Digital airbon photogrammetry a new tool for quantitiative remote sensing? a state-of-the-art review on radiometric aspects of digital photogrammetric images. Remote Sensing 1(3), pp. 577-605.

Honkavaara, E., Hakala, T., Markelin, L., Rosnell, T., Saari, H. and Mäkynen, J., 2012a. A process for radiometric correction of UAV image blocks. Photogrammetrie Fernerkundung Geoinformation 2012(2), pp. 115-127.

Honkavaara, E., Markelin, L., Rosnell, T. and Nurminen, K., 2012b. Influence of solar elevation in radiometric and geometric performance of multispectral photogrammetry. ISPRS Journal of Photogrammetry \& Remote Sensing 67, pp. 13-26.

Olsen, D., Dou, C., Zhang, X., Hu, L., Kim, H. and Hildum, E., 2010. Radiometric calibration for agcam. Remote Sensing 2, pp. 464-477.

Richter, R. and Schläpfer, D., 2002. Geo-atmospheric processing of airbone imaging spectrometry data. part 2: Atmospheric/topographic correction. International Journal of Remote Sensing 23(13), pp. 2631-2649.

Zheng, Y., Lin, S., Kambhamettu, C., Yu, J. and Kang, S. B., 2009. Single-image vignetting correction. Pattern Analysis and Machine Intelligence, IEEE Transactions on 31(12), pp. 22432256. 